\title{
CORRESPONDENCIA
}

\section{Estudio de la demanda de electromiografía en un hospital general básico}

La cuantificación de la demanda asistencial es esencial en la gestión de la asistencia neurológica, tanto por lo que respecta a la evaluación de la idoneidad de la utilización de los recursos ya destinados a cubrir unas determinadas necesidades asistenciales, como en lo referente a la planificación de la adjudicación de nuevos recursos [1]. Aunque disponemos de abundante información de calidad en lo que atañe a la vertiente puramente clínica de asistencia neurológica [2-6], no hemos encontrado referencias en la literatura sobre la demanda que la exploración de electromiografía (EMG) tiene en un hospital general básico. En el momento de organizar la actividad del laboratorio de EMG no disponíamos, pues, de una estimación fiable sobre la demanda que necesitaríamos atender. Tampoco disponemos de datos que nos orienten sobre la adecuación de la demanda de los laboratorios de EMG de los hospitales generales básicos, lo que dificulta la evaluación de intervenciones encaminadas a corregir su posible exceso [1]. Con el objeto de reorganizar la actividad asistencial en nuestro centro sobre la base de parámetros objetivos, hemos diseñado un estudio prospectivo encaminado, por una parte, a cuantificar la frecuentación del laboratorio de EMG y, por otra, a analizar desde una óptica cualitativa la adecuación de la demanda.

Por lo tanto, los objetivos del trabajo han sido, por un lado, cuantificar la demanda de EMG de forma prospectiva y, por otro, analizar la adecuación de dicha demanda.

El Hospital de Viladecans es un hospital general básico situado en el Àrea de Salut 5 de la Costa de Ponent, en la provincia de Barcelona, que presta asistencia sanitaria a una población

Tabla. Diagnósticos tras exploración electromiográfica.

\begin{tabular}{lrr}
\hline & $n$ & $\%$ \\
\hline Síndrome del túnel del carpo & 369 & 34,8 \\
\hline Radiculopatía lumbosacra & 86 & 8,1 \\
\hline Polineuropatía/mononeuritis & 47 & 4,4 \\
\hline Neuropatía cubital & 36 & 3,4 \\
\hline Radiculopatía cervical & 19 & 1,8 \\
\hline Parálisis facial periférica & 12 & 1,1 \\
\hline Plexopatía braquial & 9 & 0,8 \\
\hline Neuropatía ciático poplíteo externo & 6 & 0,6 \\
\hline Neuropatía femorocutáneo & 6 & 0,6 \\
\hline Enfermedad motoneurona & 5 & 0,5 \\
\hline Miopatías/distrofias & 5 & 0,5 \\
musculares o miotónicas & & \\
\hline Síndrome de Guillain-Barré & 2 & 0,2 \\
\hline Otros & 15 & 1,4 \\
\hline Sin alteraciones & 441 & 41,6 \\
\hline Total & 1.058 & 100
\end{tabular}

$n=$ número de pacientes. \%: proporción del diagnóstico sobre el total de exploraciones. de 144.000 habitantes. Tiene como centro de referencia el Hospital Universitario de Bellvitge. Cuenta con 100 camas para pacientes ingresados. La asistencia neurológica se realizaba en el momento del estudio con un neurólogo en dedicación completa y un refuerzo de 20 horas a la semana. Se realizaba consulta de EMG dos días a la semana para cubrir la demanda generada por los facultativos del propio hospital. En el momento de realizar el estudio se ofertaban únicamente técnicas electromiográficas básicas (electroneurografía, EMG, estudio de la onda F, estimulación repetitiva y estudio del reflejo del parpadeo). El estudio de fibra única se derivaba al Hospital Universitario de Bellvitge. A lo largo de 18 meses se recogieron de forma prospectiva, en una base de datos creada a tal efecto, las variables relativas a todos los pacientes a los que se les realizó estudio electromiográfico. En dicha base de datos se incluyó el motivo de consulta -no se podía considerar la confirmación de la normalidad como motivo de consulta- y servicio de procedencia. A todo paciente, antes de iniciar la EMG, se le realizó una anamnesis y exploración neurológica, y se introdujo directamente en la base de datos la orientación diagnóstica resultante. Hasta este momento el neurólogo era ciego respecto al motivo de consulta del médico remitente. Posteriormente, se realizó el EMG y se introdujo en la base de datos el diagnóstico electromiográfico. Para la comparación de proporciones se utilizó el test estadístico de chi al cuadrado $\left(\chi^{2}\right)$.

Se realizaron durante el período de estudio 1.058 EMG. Teniendo en cuenta nuestra población de referencia, la demanda hospitalaria de EMG calculada sería de 4,8/1.000 habitantes/año. Se obtuvieron un total de 24 diagnósticos diferentes después de la realización de la prueba. El EMG resultó normal en el $41 \%$ de las exploraciones (441 pacientes). Por otro lado, en 50 pacientes el EMG permitió el diagnóstico de una entidad previamente no sospechada por el médico remitente. Tres especialidades acumularon más del 95\% de la demanda de EMG: Cirugía Ortopédica y Traumatología (COT) (68\%), Reumatología (REU) (17\%) y Neurología (NRL) $(10,6 \%)$. COT solicitó la prueba por un total de 20 diagnósticos de sospecha diferentes, REU por 10 y NRL por 23. Por patologías, el diagnóstico que más frecuentemente motivó la solicitud de EMG fue el síndrome del túnel del carpo (42\%), seguido por radiculopatía lumbosacra $(20 \%)$ y cervical (12\%), neuropatía cubital $(7 \%)$ y polineuropatía $(6 \%)$. Estos cinco motivos de consulta constituyeron más del $80 \%$ de la demanda de exploración. Los diagnósticos emitidos después de la realización del EMG se reflejan en la tabla. El diagnóstico electromiográfico más frecuentemente emitido fue el del síndrome del túnel del carpo $(38,4 \%)$, seguido de radiculopatía lumbosacra $(8,1 \%)$, polineuropatía $(4,4 \%)$ y neuropatía cubital $(3,4 \%)$. La proporción de EMG sin alteraciones en función del tipo de diagnóstico de sospecha fue similar en todas las patologías (alrededor del $40 \%$ ), a excepción de la radiculopatía cervical, en la que sólo en 15 de 125 ocasiones (12\%) la exploración EMG confirmó la sospecha clí- nica. Por especialidades, COT presentó una proporción de EMG con diagnóstico de "normalidad' significativamente superior al resto de especialidades ( 45 frente a $40 \%$ de REU y $30 \%$ de NRL; $p<0,05$ de COT frente a NRL). Dicha diferencia fue a expensas, precisamente, de los EMG realizados ante sospecha de radiculopatía cervical.

En un 54\% de los pacientes la exploración confirmó la sospecha diagnóstica que motivó la petición de EMG (50\% de los solicitados por COT, $57 \%$ por REU, $65 \%$ por NRL). El neurólogo que realizó la exploración previa al EMG consideró que éste se indicaba en el $73 \%$ de los pacientes, coincidiendo con la misma orientación diagnóstica del médico que refería el paciente en el $68 \%$ de las ocasiones. En 22 de los 294 pacientes $(7,4 \%)$ en los que el neurólogo que realizaba la prueba consideraba que ésta sería normal, se detectó patología (2 polineuropatías, 2 neuropatías cubitales y 18 síndrome del túnel del carpo, todas de leve intensidad).

La demanda de asistencia neurológica ambulatoria se ha cifrado entre 10 y 27 pacientes/ 1.000 habitantes y año [3-6]. Consideramos que la cifra sobre la demanda de EMG hallada en nuestro trabajo $(4,8 / 1.000$ habitantes y año) es un dato interesante a la hora de calcular las necesidades que, para una correcta atención neurológica, tiene un hospital general. No hemos hallado en la literatura referencias sobre el origen de la demanda asistencial en una consulta de EMG. En nuestro caso concreto, la mayoría de solicitudes de exploración proviene de la consulta de COT. En nuestro centro existe un interés especial por parte de traumatólogos en el traumatismo de nervio y su reparación, lo que puede contribuir a una elevada demanda. En otro estudio realizado en nuestro país, el $44,17 \%$ de las solicitudes provenían del servicio de NRL -aunque dicho estudio incluye la realización de potenciales evocados y se realizó en un hospital universitario-. En cuanto al tipo de patología observada en la consulta, ésta es el resultado del propio origen de la demanda (COT y REU, principalmente). En la literatura lo que se recoge sobre proporción de diagnósticos es diferente a lo encontrado en nuestro centro, predominando la consulta por sospecha de miopatía, posiblemente porque el ámbito de asistencia sea más especializado [7]. En el estudio de Sureda et al [8], sin embargo, la patología más frecuente fue también el síndrome del túnel del carpo. En cuanto a la adecuación de la demanda, Mondelli et al, en 1999 [9], publicaron un trabajo en el que el EMG confirmaba el diagnóstico clínico en el $45 \%$ de las ocasiones. En nuestro caso, dicha confirmación del diagnóstico de sospecha se alcanza en el 54\%. La concordancia del diagnóstico clínico entre el médico que deriva el paciente y el neurólogo que realiza la exploración se consiguió en el $68 \%$ de los casos, proporción que supera a la obtenida en el estudio italiano $(57 \%)$. Estas diferencias pueden deberse, precisamente, a que en nuestro caso la derivación procede del propio centro y en su mayor parte de COT, REU y NRL, especialidades con las que Mondelli et al [9] también 
encuentran una mayor concordancia diagnóstica. El médico que realizaba el EMG consideró en el $73 \%$ de las ocasiones que la exploración se indicaba -lo que deja un $27 \%$ de ocasiones en los que el EMG podría considerarse solicitado para 'confirmar normalidad'-. En el estudio italiano se concluye que el EMG se indicaba a criterio del electromiografista en el $77 \%$ de las ocasiones [9]. Un dato a destacar es el amplio número de diagnósticos diferentes que se obtuvieron tras la realización de la exploración, lo que da idea de la complejidad de dicha consulta.

En base a los resultados obtenidos, consideramos que la demanda de EMG en nuestro hospital es adecuada, y sorprende incluso la escasa proporción de exploraciones que se podrían considerar solicitadas para 'confirmar normalidad'. Por todo ello, creemos que la implementación de laboratorios de EMG en los hospitales generales básicos contribuye a aumentar la eficiencia en el tratamiento de patologías prevalentes, disminuyendo la carga asistencial de los hospitales de alta tecnología, con la ventaja añadida de la proximidad del usuario.

A. Martínez-Yélamos ${ }^{a}$, M. Admetlla-Falguera ${ }^{\text {b }}$, M. Huerta-Villanueva a ${ }^{\text {, M. Jato-de Evan }}{ }^{\text {a }}$, S. Martínez-Yélamos ${ }^{\mathrm{c}}$

Aceptado: 17.03.05.

${ }^{a}$ Servicio de Neurología. ${ }^{b}$ Servicio de Medicina Interna. Hospital de Viladecans. Viladecans, Barcelona. ${ }^{c}$ Servicio de Neurología. Hospital Universitari de Bellvitge. L'Hospitalet de Llobregat, Barcelona, España.

Correspondencia: Dr. Antonio Martínez-Yélamos. Servicio de Neurología. Hospital de Viladecans. Avda de Gavà, 38. E-08840 Viladecans. E-mail: antonio martinezy@gencat.net

\section{BIBLIOGRAFÍA}

1. Martín R, Matías-Guiu J. Modelos de asistencia neurológica. In Matías-Guiu J, Láinez, JM, eds. Gestión sanitaria y asistencia neurológica. Barcelona: Prous; 1994. p. 13-21.

2. Sempere AP, Mola S, Medrano V, Esguevillas T, Costa C, Salazar V, et al. Epidemiología descriptiva de la asistencia neurológica ambulatoria en el Área Vega Baja (Alicante). Rev Neurol 2002; 35: 822-6.

3. Batalla X. Neurología extrahospitalaria: Análisis descriptivo de una consulta en el Baix Llobregat. Rev Neurol 1997; 25: 1546-50.

4. Morera-Guitart J. Estudio transversal multicéntrico de la asistencia neurológica extrahospitalaria en la Comunidad Valenciana. Rev Neurol 1998; 27: 438-46.

5. Pérez-Carmona N, Sánchez-Pérez RM, Abellán-Miralles I, Díaz-Marín C. Asistencia neurológica ambulatoria en el área de la Marina Baixa, Alicante. Rev Neurol 2004; 39: 607-13.

6. González-Menacho J, Olivé-Plana JM. Epidemiología de la patología neurológica ambulatoria en el Baix Camp (Tarragona) durante 1999. Neurología 2001; 16: 154-62.

7. Dumitru D. The electrodiagnostic medicine consultation: report generation and approach. In Dumitru D, ed. Electrodiagnostic medicine. Philadelphia: Hanley \& Belfus; 1995. p. 387-412.
8. Sureda B, Miralles F, Moreno A, Calles C, Molina F, Gorospe G. Sistema organizativo de la electromiografía en el marco de un servicio de Neurología (Unidad de Patología Neuromuscular-Electrodiagnóstico). Neurología 2001; 16: 514.

9. Mondelli, M, Giacchi M, Federico A. Requests for electromyography from general practitioners and specialists. Critical evaluation. Ital Neurol Sci 1998; 19: 195-203.

\section{Impulso bioeléctrico, cuantificaciones sinápticas y de circuitos cerebrales, origen de la vida y composición principal de las neuronas}

Hemos estudiado el interesantísimo artículo de Pascual-Garvi et al, que, aunque centrado especialmente en la barrera hematoencefálica [1], aborda aspectos particulares de las neuronas, los cuales se asumen de ciertas referencias citadas en la bibliografía -por lo que creemos no es error directo de estos autores, sino de las fuentes asumidas-; también se hace referencia a un aspecto sobre la evolución, para el cual no hay cita bibliográfica de base -en este caso es un error de apreciación de estos autores-. Por estos detalles citados, presentamos algunos aportaciones como puntos de vista contrarios, además de ciertas dudas que nos suscitan algunas de sus anotaciones tan puntuales hechas entorno al cerebro humano, para las que actuamos por inferencia lógica, más no empírica; aunque creemos que esta deficiencia se debe a la falta de observación, que es más segura que la basada en la deducción de principios racionales, en especial si se establece a través de una muy cuidadosa observación mediante la investigación experimental.

Las anotaciones a contrastar sobre el cerebro son:

1. Posee neuronas con capacidad de respuesta eléctrica.

2. Sus neuronas se interconectan, por término medio, con otras 10.000 .

3. El número posible estimado de circuitos y combinaciones de conexiones en un único cerebro sobrepasa con mucho el número de átomos existente en todo el universo.

4. Se han necesitado más de 4.000 millones de años de evolución de la vida sobre el planeta Tierra para dar lugar al cerebro humano.

5. El cerebro humano está compuesto principalmente por lípidos [1].

Con respecto a estas anotaciones, y según el orden numérico presentado, exponemos seguidamente nuestras consideraciones que apuntan a lo constructivo:

1. Pensamos que las neuronas no poseen capacidad de respuesta eléctrica y mejor sería considerar una respuesta bioeléctrica, aunque el sensor que se utilice para el registro y amplificación de la señal lo haga en términos eléctricos. A pesar de que el término eléctrico es muy usado en la literatura sobre fisiología del siste- ma nervioso, posiblemente se aplique erradamente como una homología y es que se trata de una analogía [2], ya que es más bien una comparación entre dos sistemas o dominios de conocimientos distintos que mantienen una cierta relación de semejanza entre sí [3], el biológico y el físico (eléctrico). Varias razones nos llevan a considerar esta forma de razonamiento:

- Los electrones que llevan una señal eléctrica tienen una velocidad de arrastre calculada mediante la formula $V d=1 / n e A$, donde $n$ es el número de electrones libres por unidad de volumen del conductor, $e$ equivale a la carga del electrón, que es de 1,6 6 $10^{-19}$ culombios, y $A$ corresponde al área de sección transversal del alambre. Por tanto, en la velocidad de arrastre, $V d=2,46 \times$ $10^{-4} \mathrm{~m} / \mathrm{s}$, los electrones tardan 68 minutos en recorrer un solo metro de cable de cobre y el campo eléctrico viaja a una velocidad aproximada a la de la luz [4]. Algo muy distinto sucede con el impulso nervioso, el cual se conduce a una velocidad mucho menor que un campo eléctrico; por ejemplo, puede alcanzar hasta los $120 \mathrm{~m} / \mathrm{s}$ en fibras gruesas de carácter mielínico [5] debido al flujo de iones por canales proteicos de la membrana celular, a manera de un impulso bioiónico.

- Un cable eléctrico presenta una nube electrónica comunitaria en la que los electrones fluyen libremente a través del metal [6], donde la carga eléctrica es un exceso o una falta de electrones [7]; mientras que por el axón de las neuronas fluyen iones en forma saltatoria y restringida a canales proteicos específicos ubicados en los espacios internodales (de Ranvier), en los que, por ejemplo, los canales de $\mathrm{Na}^{+}$son muy abundantes, unos 10.000 por $\mathrm{mm}^{2}$ [8].

Se concluye, de momento, que la analogía de la respuesta eléctrica no es equivalente a la bioeléctrica, sino una comparación entre dominios esencialmente diferentes y que pretender establecer identidades entre estos dominios acabará ocasionando problemas; creemos, en consecuencia, y plantando esta semilla de duda razonable, que el término que se debe aplicar en los eventos biológicos es el bioeléctrico y no el eléctrico.

2. Es dudoso que las neuronas del cerebro se interconecten con otras 10.000 más en término medio, ya que diversos autores de renombre en el área de la neurociencia presentan valores dispares; así, por ejemplo:

- Una neurona realiza un promedio de 1.000 conexiones sinápticas y todavía recibe más, quizás unas 10.000 conexiones [9]

- Las neuronas del cerebro conectan con 1.000 sinapsis $[10,11]$.

- En promedio, cada neurona se divide para formar 2.000 terminaciones sinápticas, aunque se calcula que el número de botones sinápticos adheridos a una motoneurona espinal son cerca de 10.000 , de los cuales 2.000 se dan en el soma y 8.000 en las dendritas $[9,12]$.

- El valor de 10.000 sinapsis se ha asignado sin tener en cuenta ninguna diferenciación entre las neuronas, valor que se ha extrapo- 